\title{
Efectos del retardo en el control de lazo cerrado de plantas sobreamortiguadas
}

\author{
Recibido: 23/02/12 Aceptado: 20/06/12
}

Bruno Vargas Tamani ${ }^{1}$

\begin{abstract}
RESUMEN
El presente trabajo presenta un estudio del efecto del retardo en el desempeño de los sistemas de lazo cerrado de control de plantas industriales que pueden modelarse como de segundo orden sobreamortiguadas, en las cuales no se ha considerado el retardo en el diseño del controlador. Se eligen los tipos de plantas representativas de segundo orden y se diseñan para cada una de ellas como controlador, al clásico controlador proporcional integral derivativo (PID). Se experimenta con plantas de prueba a las cuales previamente se realizan procedimientos de identificación analógica y luego son controladas por los PID correspondientes incorporando retardos a las plantas no consideradas en el diseño. Se realizan las pruebas correspondientes para obtener el desempeño de lazo cerrado midiéndose los parámetros de respuesta dinámica a la salida de la planta.
\end{abstract}

Palabras claves: sistemas de lazo cerrado, controlador pid, identificación de sistemas, diseño de controladores, respuesta dinámica.

EFFECT OF DELAY IN THE CLOSED LOOP CONTROL ON PLANTS DAMPED

\section{ABSTRACT}

This paper presents a study of the effect of delay in the performance of closed loop systems control plants that can be modeled as second order sobreamortiguadas, in which were not considered the delay in the controller design. They choose the types of plants representative of the second order and are designed for each driver to the classic controller as proportional integral derivative (PID). Experienced with test plants which are conducted previously analog and identification procedures are then controlled by the PID incorporating delays corresponding to plants is not considered in the design. Tests are performed to obtain the corresponding closed-loop performance parameters measured dynamic response to the output of the plant.

Keywords: closed loop systems, pid controller, system identification, controller design, dynamic response.

\section{INTRODUCCIÓN}

Los tiempos de retardo o tiempos muertos constituyen una parte fundamental de la dinámica de muchos procesos industriales, siendo una limitante para conseguir un control adecuado; por ello, es importante tomarlo en cuenta durante el diseño del controlador del proceso ya que son una fuente de inestabilidad en los lazos de control. El tiempo muerto, es el que transcurre desde el instante en que se produce un cambio en una variable de entrada al proceso hasta el instante en que el efecto de dicha variación comienza a observarse en la variable de salida.

Normalmente, se debe al tiempo que tarda un fluido en circular de un punto a otro a través de tuberías (también conocido como retardo "distancia-velocidad"), a una mezcla imperfecta en un equipo tipo tanque agitado o al sistema de medida de la variable controlada. Los procesos que presentan un tiempo muerto significativo frente a la constante de tiempo dominante son difíciles de controlar con un bucle de realimentación simple tipo PID. La razón fundamental se debe a que el atraso de fase que introduce el tiempo muerto del proceso (fase negativa y grande incluso a bajas frecuencias) hace necesario "desajustar" el controlador (ganancia proporcional baja y tiempo integral grande) para poder asegurar la estabilidad del sistema en bucle cerrado. Este "desajuste" del controlador provoca que el sistema en bucle cerrado de este tipo de procesos presente un comportamiento lento y bajo rechazo a perturbaciones. Por ello, cuando la relación entre el tiempo muerto del proceso y la constante de tiempo dominante sea mayor que 2 es aconsejable utilizar algoritmos específicos de compensación del tiempo muerto para controlar el proceso [1]. Una amplia gama de controladores de procesos que se utilizan en la industria ofrecen como principal la estrategia de control proporcional integral derivativo o comúnmente llamado controlador PID, la cual en muchos casos presenta la dificultad de ajuste eficiente de estos controladores. El procedimiento de sintonía del controlador PID es tediosa y requiere mucho tiempo, el desempeño del sistema de lazo cerrado principalmente depende de la experiencia y del conocimiento que el ingeniero encargado tiene del proceso. Se reconoce en la práctica que muchos lazos cerrados de control industrial están pobremente sintonizados. Las técnicas de sintonización automática, por lo tanto, llaman la atención cada vez más de los investigadores y de los ingenieros en ejercicio [2].

1 Magíster en Ingeniería de Control. Docente Facultad de Ingeniería Electrónica y Eléctrica, Universidad Nacional Mayor de San Marcos, Lima, Perú. E-mail: bvargast@unmsm.edu.pe. 


\section{SISTEMAS DE SEGUNDO ORDEN SOBREA- MORTIGUADOS REPRESENTATIVOS PARA UN AMPLIO RANGO DE VALORES DE RELACIÓN DE AMORTIGUAMIENTO}

Una planta con entrada $\mathrm{u}(\mathrm{t})$ y salida $\mathrm{y}(\mathrm{t})$ (con transformadas de Laplace $U(\mathrm{~s})$ y $Y(\mathrm{~s})$ respectivamente) que representa un sistema de segundo orden tiene la siguiente función de transferencia [3] :

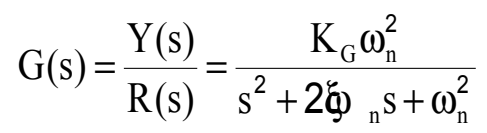

Los parámetros que caracterizan a un sistema de segundo orden son: su ganancia $\mathrm{K}_{\mathrm{G}}$, su relación de amortiguamiento $\xi$ y la frecuencia natural no amortiguada $\omega_{n}$. El término $\xi \omega_{n}=\sigma$ es llamado atenuación.

De acuerdo al valor de la relación de amortiguamiento se clasifican los sistemas de segundo orden, para $\xi>1$ el sistema se denomina de segundo orden sobreamortiguado. Este sistema está caracterizado por dos constantes de tiempo $\mathrm{T}_{1}$ y $_{\mathrm{T}}$ (asumimos la condición $\mathrm{T}_{1}>\mathrm{T}_{2}$ ).

La ecuación (1) para un sistema sobreamortiguado se puede representar por:

$$
\mathrm{G}(\mathrm{s})=\frac{\mathrm{K}_{\mathrm{G}}}{\left(\tau_{1} \mathrm{~s}+1 \mathrm{1} \tau_{2} \mathrm{~s}+1\right)}
$$

Definimos $\rho$ como la relación entre las constantes de tiempo $\mathrm{T}_{2}$ У $_{1}$ :

$$
\rho=\frac{\tau_{2}}{\tau_{1}}(3)
$$

Con (3) en (2) el sistema sobreamortiguado se representa por :

$$
\mathrm{G}(\mathrm{s})=\frac{\mathrm{K}_{\mathrm{G}}}{\left(\tau_{1} \mathrm{~s}+1 \gamma \mathrm{p}_{1} \mathrm{~s}+1\right)}
$$

Para una entrada escalón de amplitud unitaria la respuesta del sistema sobreamortiguado de ganancia $\mathrm{K}_{\mathrm{G}}=1$, está dada por [4] :

$$
y(t)=1+\frac{\rho}{1-\rho} e^{-t / \rho} 1-\frac{1}{1-\rho} e^{-t / \tau_{1}}
$$

La relación de amplitudes de los componentes transitorios que corresponden a la constante de tiem- po más rápida $\mathrm{T}_{2}$ respecto a la constante de tiempo más larga $\mathrm{T}_{1}$, es igual a $\rho$.

La Figura 1, muestra un juego de respuestas al escalón unitario de sistemas sobreamortiguados con ganancia unitaria para diferentes valores de $\rho$, considerando una constante de tiempo normalizada $\mathrm{T}_{1}=1$.

Figura 1. Respuestas al escalón unitario de sistemas sobreamortiguados para diferentes valores de $\rho$.

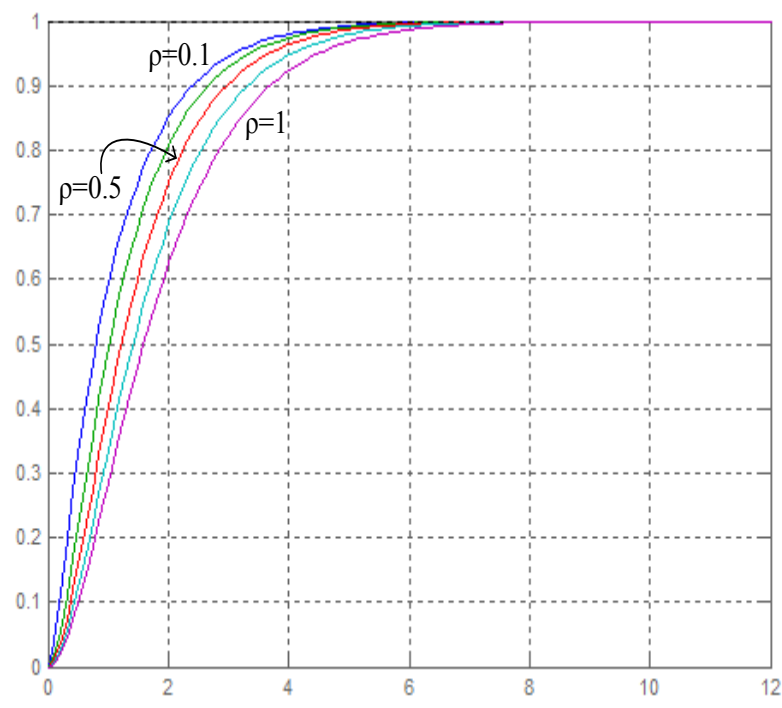

Cuando $\rho$ es muy pequeño, de acuerdo a (5) la constante de tiempo $\mathrm{T}_{2}$ produce una respuesta transitoria más rápidamente decreciente que el transitorio producido por la constante de tiempo $\mathrm{T}_{1}$. Con esto se podría despreciar la constante de tiempo $\mathrm{T}_{2}$ y el sistema se comportará como uno equivalente de primer orden.

Cuando $\rho$ es 1 se trata de un sistema críticamente amortiguado $(\xi=1)$.

Un sistema representativo de los sistemas sobreamortiguados para un rango $0<\rho<1$, puede ser el que corresponde a la respuesta central de la figura 1 (respuesta intermedia entre la respuesta equivalente de primer orden y la respuesta de un sistema críticamente amortiguado) para el cual el valor de $\rho$ es igual a 0.5 .

\section{DISEÑO DE CONTROLADORES PID PARA LAS PLANTAS REPRESENTATIVAS SOBREAMORTI- GUADAS}

A. Diseño de controlador PID para una planta representativa de segundo orden sobreamortiguada con $\rho=0.5$. 
La Figura 2 representa el sistema de lazo cerrado para el control de la planta representativa G(s). La planta $\mathrm{G}(\mathrm{s})$ será controlada mediante un controlador con función de transferencia $\mathrm{G}_{\mathrm{C}}(\mathrm{s})$ en cascada con la planta, mediante un sistema de lazo cerrado con realimentación unitaria.

En la Figura 2, r(t) es la señal de referencia de lazo cerrado, e(t) es la señal de error que se desea anular en estado estacionario para cumplir con el objetivo que la referencia se refleje en la salida de la planta. El controlador representado por $\mathrm{G}_{\mathrm{c}}(\mathrm{s})$ en todo tiempo $t$ debe producir la señal de control $u(t)$ que se aplica a la planta para asegurar el objetivo de control.

Figura 2. Diagrama de bloques del sistema de lazo cerrado para controlar la salida de la planta $\mathrm{y}(\mathrm{t})$.

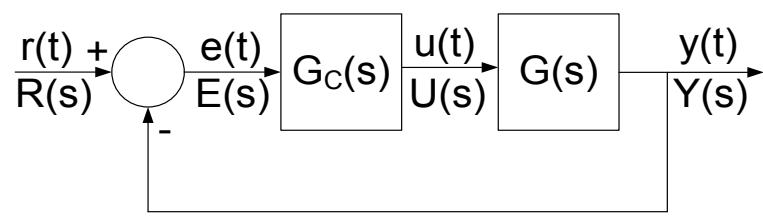

Para una planta de segundo orden sobreamortiguada, se cumple que los dos polos $s_{1}$ y $s_{2}$ de esa planta de acuerdo a (2), se relacionan con las constantes de tiempo $\mathrm{T}_{1} \mathrm{y}_{2}$ a través de :

$$
\begin{aligned}
& s_{1}=-\frac{1}{\tau_{1}}(6) \\
& s_{2}=-\frac{1}{\tau_{2}}(7)
\end{aligned}
$$

Con el valor representativo $\rho=0.5$ y de acuerdo a (3), se cumple:

$$
s_{2}=2 s_{1}(8)
$$

Si consideramos que el sistema de lazo cerrado debe estar manejado en su respuesta transitoria por un par de polos dominantes subamortiguados con amortiguamento $\xi_{L C}=0.5$, entonces los polos de lazo cerrado dominantes $\mathrm{s}_{\mathrm{LC} 1}$ y $\mathrm{s}_{\mathrm{LC} 2}$ (donde ambos son conjugados), deben ubicarse sobre la línea que sale del origen con un ángulo $\alpha$ entre el eje real negativo y dicha línea, la cual cumple:

$$
\cos \alpha=\xi_{C}=0.5
$$

Con lo cual :

$$
\alpha=\cos ^{-1}(0.5)=0
$$

Para asegurar la condición estacionaria se debe contar con un componente integral en el controlador $\mathrm{G}_{\mathrm{C}}(\mathrm{s})$. Ese componente integral asegura la anulación del error en estado estacionario para sistemas estables de lazo cerrado, para entradas de referencia y de perturbación constantes. El retraso de fase que produce el integrador se debe compensar agregando un par de ceros iguales. Con lo cual el controlador tendrá la estructura PID dada por:

$$
\mathrm{G}_{\mathrm{C}}(\mathrm{s})=\mathrm{K} \frac{(\mathrm{s}-\mathrm{a})^{2}}{\mathrm{~s}}
$$

La Figura 3, muestra la distribución de polos y ceros de la trayectoria directa formada por el controlador propuesto y la planta, además del polo dominante de lazo cerrado en el tercer cuadrante elegido.

A partir de la condición de fase del lugar geométrico de las raíces [5] y en base a las relaciones geométricas mostradas en la Figura 3 , el valor de a se obtiene como:

$$
a=3 s_{1}
$$

Utilizando la condición del módulo del lugar geométrico de las raíces [5], se obtiene la ganancia $\mathrm{K}$ de control como:

$$
K=\frac{1.1667}{K_{G}\left|S_{1}\right|}
$$

Por tanto, al controlador propuesto diseñado a partir de (12) y (13) reemplazado en (11) le corresponde la siguiente función de transferencia:

$$
G_{C}(s)=\frac{1.1667}{K_{G}\left|s_{1}\right|} \frac{\left(s-3 s_{1}\right)^{2}}{s}
$$


Figura 3. Vectores y ángulos trazados desde los polos y ceros de la trayectoria directa $G_{L G R}(s)$ al polo deseado $\mathrm{S}_{\mathrm{LC} 1}$.

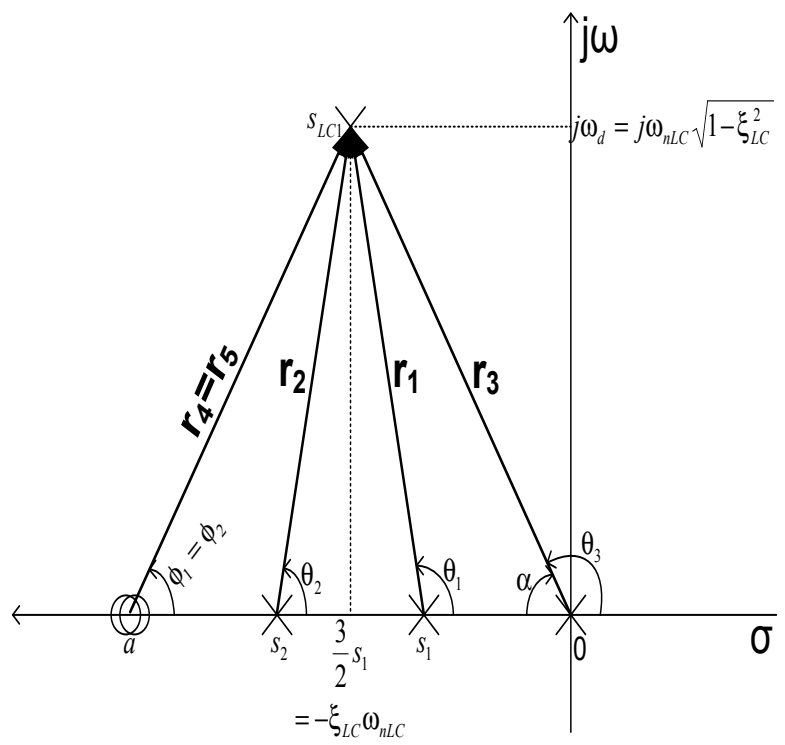

B. Diseño de controlador PID para una planta críticamente amortiguada.

Para una planta representativa de segundo orden críticamente amortiguada, se presenta un amortiguamiento de $\xi=1$. Esa planta la caracterizan dos constantes de tiempo $\mathrm{T}_{1}$ y $\mathrm{T}_{2}$, con la condición $\mathrm{T}_{1}=\mathrm{T}_{2}$. La ganancia de la planta es $\mathrm{K}_{\mathrm{G}}$, el modelo de la planta, según (2), presenta polos iguales y se cumple :

$$
\tau_{1}=\tau_{2}=-\frac{1}{s_{1}}
$$

Los polos dominantes se eligen con las consideraciones dadas en (9) y (10). Por las razones dadas en III. A., el controlador PID está representado por (11).

La Figura 4, muestra la distribución de polos y ceros de la trayectoria directa formada por el controlador propuesto y la planta, además del polo dominante de lazo cerrado en el tercer cuadrante elegido.
Figura 4. Vectores y ángulos trazados desde los polos y ceros de la trayectoria directa $\mathrm{G}_{\mathrm{LGR}}(\mathrm{s})$ al polo deseado $\mathrm{s}_{\mathrm{LC} 1}$.

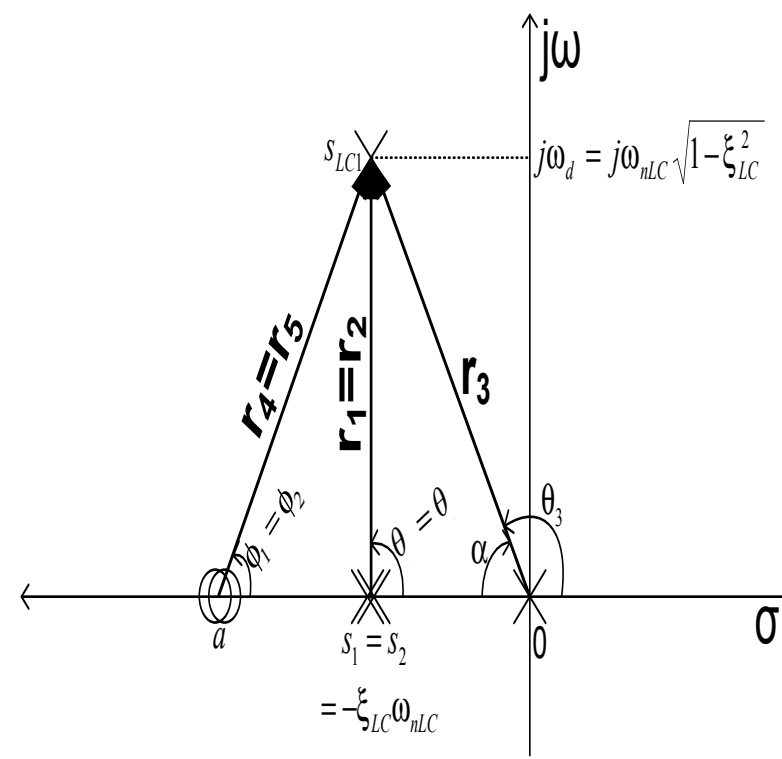

Utilizando las condiciones de fase y de módulo del lugar geométrico de las raíces, se obtiene el siguiente controlador PID:

$$
\mathrm{G}_{\mathrm{C}}(\mathrm{s})=\frac{1.5}{\mathrm{~K}_{\mathrm{G}}\left|\mathrm{s}_{1}\right|} \frac{\left(\mathrm{s}-2 \mathrm{~s}_{1}\right)^{2}}{\mathrm{~s}}
$$

C. Obtención de PID realizable agregando un polo adicional igual a tres veces la parte real del polo dominante.

Los controladores propuestos dados por (14) y (16) presentan estructuras de función de transferencia no realizables. Para obtener un controlador PID que se pueda implementar, debemos modificar la representación de los controladores diseñados y lograr su realizabilidad. Se propone agregar un polo adicional $\mathrm{s}_{\mathrm{ad}}$ alejado a la izquierda de la zona de incidencia del polo dominante, de tal manera que el controlador realizable tenga la siguiente función de transferencia:

$$
\mathrm{G}_{\text {Creal }}(\mathrm{s})=\mathrm{G}_{\mathrm{C}}(\mathrm{s}) \mathrm{G}_{\mathrm{Cad}}(\mathrm{s})
$$


Donde $\mathrm{G}_{\mathrm{C}}(\mathrm{s})$ representa a cada uno de los controladores diseñados y $\mathrm{G}_{\mathrm{Cad}}(\mathrm{s})$ representa la función de transferencia del componente adicional que permitirá que el conjunto representado por (17) sea realizable. El componente $\mathrm{G}_{\mathrm{Cad}}(\mathrm{s})$ que se adicionará debe presentar un polo adicional, por tanto, se sugiere la siguiente función :

$$
G_{C a d}(s)=\frac{-s_{d}}{\left(s-s_{d}\right)}
$$

La ganancia de $\mathrm{G}_{\mathrm{Cad}}(\mathrm{s})$ será unitaria. El polo adicionado en $\mathrm{s}_{\text {ad }}$ debe ser ubicado alejado de la zona dominante de lazo cerrado, ese polo será un número $\mathrm{n}$ de veces la parte real del polo dominante, así :

$$
s_{d l}=n \operatorname{Re}\left(s_{C} 1\right)
$$

Considerando $n=10$ y (19) en (18) y en (17), a partir del controlador PID representado por (14) se obtiene el siguiente controlador PID realizable :

$$
\mathrm{G}_{\text {Creal }}(\mathrm{s})=\frac{7.5}{\mathrm{~K}_{\mathrm{G}}} \frac{\left(\mathrm{s}-3 \mathrm{~s}_{1}\right)^{2}}{\mathrm{~s}\left(\mathrm{~s}-5 \mathrm{~s}_{1}\right)}
$$

y el controlador PID representado por (16) realizable será el siguiente :

$$
\mathrm{G}_{\text {Creal }}(\mathrm{s})=\frac{5}{\mathrm{~K}_{\mathrm{G}}} \frac{\left(\mathrm{s}-2 \mathrm{~s}_{1}\right)^{2}}{\mathrm{~s}\left(\mathrm{~s}-\mathbb{0} \mathrm{s}_{1}\right)}
$$

\section{ANÁLISIS DEL EFECTO DEL RETARDO DE LA PLANTA EN EL DESEMPEÑO DE LAZO CERRA- DO DE LAS PLANTAS REPRESENTATIVAS SO- BREAMORTIGUADAS}

A. Análisis del efecto del retardo en el desempeño de lazo cerrado de la planta representativa sobreamortiguada con $\rho=0.5$

Consideramos la planta representativa de segundo orden sobreamortiguada con $\rho=0.5$ con ganancia unitaria $\mathrm{K}_{\mathrm{G}}$ y constante de tiempo mayor $\mathrm{T}_{1}$ también unitaria reemplazando en (4) se obtiene la siguiente función de transferencia de la planta :

$$
G(s)=\frac{2}{s^{2}+3 s+2}
$$

El controlador realizable sugerido para esta planta está representado por (20). Considerando en (20) $\mathrm{s}_{1}=1 / \mathrm{T}_{1}=-1$, el controlador realizable para esta planta será el siguiente:

$$
\mathrm{G}_{\text {Creal }}(\mathrm{s})=7.5 \frac{(\mathrm{s}+3)^{2}}{\mathrm{~s}(\mathrm{~s}+5)}
$$

El sistema de lazo cerrado de acuerdo a la Figura 2, considerando (22) y (23), será analizado en términos del efecto en la estabilidad de lazo cerrado del tiempo de retardo $\mathrm{m}$ que presenta la planta y que no fue considerado en el diseño del controlador PID. (Para sistemas de lazo cerrado estables, la respuesta estacionaria está asegurada por la presencia del componente integral en el controlador). Para diferentes relaciones del tiempo de retardo $\mathrm{m}$ a la constante de tiempo mayor de la planta $\mathrm{T}_{1}$, la Figura 5 muestra la respuesta en frecuencia de la trayectoria directa en términos de gráficos de magnitud y fase. La Tabla I, muestra cómo varían los márgenes de fase (MF), de ganancia $(M G)$ y las frecuencias de cruce de ganancia $\left(\omega_{c g}\right)$ y de fase $\left(\omega_{c f}\right)$.

Se aprecia de la Figura 5 (y de los resultados mostrados en la Tabla I) que la frecuencia de cruce de ganancia no se afecta por el aumento del retardo, pero sí se aprecia una disminución del margen de fase llegándose a situaciones inestables. Esto se confirma con la disminución del margen de ganancia.

Figura 5. Variación de la respuesta en frecuencia en magnitud y fase de la trayectoria directa por variación del retardo de la planta sobreamortiguada.

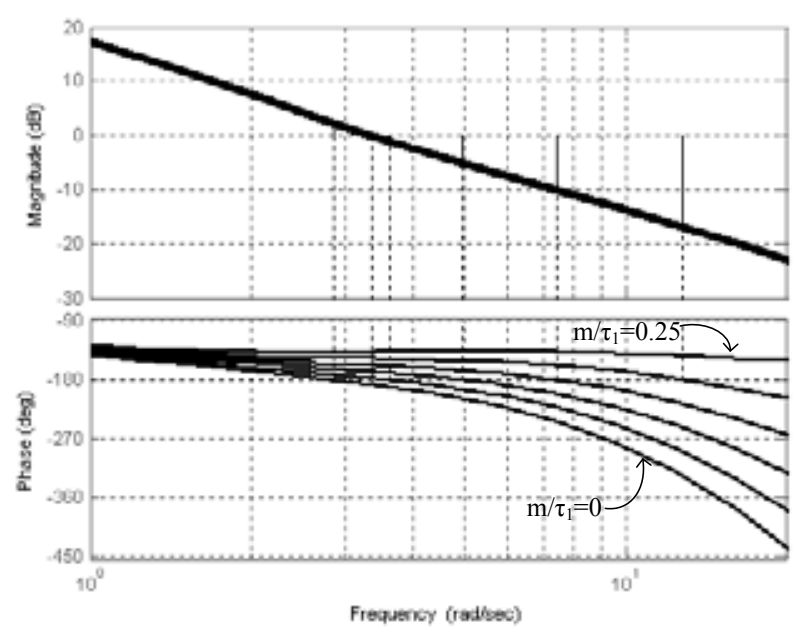


Tabla I. Márgenes de ganancia y de fase. Frecuencias de cruce de fase y de ganancia al variar el retardo de la planta sobreamortiguada

\begin{tabular}{|c|c|c|c|c|}
\hline $\mathrm{m} / \mathrm{T}_{1}$ & $\mathrm{MG}$ & $\omega_{\mathrm{cf}}$ & $\mathrm{MF}$ & $\omega_{\mathrm{cg}}$ \\
\hline 0 & $\infty$ & $\infty$ & 41.1978 & 3.3665 \\
\hline 0.05 & 16.7830 & 12.7565 & 31.5535 & 3.3665 \\
\hline 0.1 & 10.0401 & 7.4036 & 21.9092 & 3.3665 \\
\hline 0.15 & 5.1505 & 4.9719 & 12.2649 & 3.3665 \\
\hline 0.2 & 0.9996 & 3.6211 & 2.6206 & 3.3665 \\
\hline 0.25 & -2.3887 & 2.8409 & -7.0237 & 3.3665 \\
\hline 0.3 & -5.0428 & 2.3659 & -16.6680 & 3.3665 \\
\hline 0.35 & -7.1272 & 2.0539 & -26.3123 & 3.3665 \\
\hline 0.4 & -8.8138 & 1.8324 & -35.9566 & 3.3665 \\
\hline
\end{tabular}

B. Análisis del efecto del retardo en el desempeño de lazo cerrado de la planta representativa críticamente amortiguada

Se considera la planta representativa de segundo orden críticamente amortiguada con $\rho=1$, con ganancia unitaria $\mathrm{K}_{\mathrm{G}}$ y constante de tiempo mayor $\mathrm{T}_{1}$ también unitaria; reemplazando en (4) se obtiene la siguiente función de transferencia de la planta :

$$
G(s)=\frac{1}{(s+1)^{2}}
$$

El controlador realizable sugerido para esta planta está representado por (21). Considerando $s_{1}=1 / t_{1}=-$ 1 en (6), el controlador realizable para esta planta será el siguiente :

$$
\mathrm{G}_{\text {Creal }}(\mathrm{s})=5 \frac{(\mathrm{s}+2)^{2}}{\mathrm{~s}(\mathrm{~s}+\mathbb{0})}
$$

El sistema de lazo cerrado de acuerdo a la Figura 2, considerando (24) y (25), será analizado en términos del efecto en la estabilidad de lazo cerrado del tiempo de retardo $m$ que presenta la planta $y$ que no fue considerado en el diseño del controlador PID. Se considera una relación del retardo $\mathrm{m}$ a la constante de tiempo mayor de la planta $\mathrm{T}_{1}$ variable. Para diferentes relaciones del tiempo de retardo retardo $\mathrm{m}$ a la constante de tiempo mayor de la planta $\mathrm{T}_{1}$, la figura 6 muestra la respuesta en frecuencia de la trayectoria directa en términos de gráficos de magnitud y fase. La Tabla II, muestra cómo varían los márgenes de fase, de ganancia y las frecuencias de cruce de ganancia y de fase.
Figura 6. Variación de la respuesta en frecuencia en magnitud y fase de la trayectoria directa por variación del retardo de la planta críticamente amortiguada.

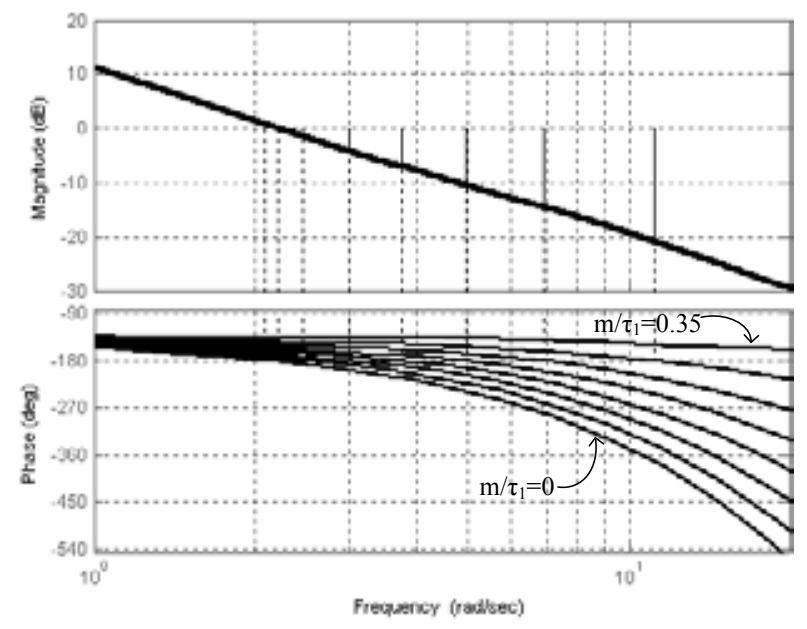

Tabla II. Márgenes de ganancia y de fase. Frecuencias de cruce de fase y de ganancia al variar el retardo de la planta críticamente amortiguada.

\begin{tabular}{|c|c|c|c|c|}
\hline $\mathrm{m} / \mathrm{T}_{1}$ & $\mathrm{MG}$ & $\omega_{\mathrm{cf}}$ & $\mathrm{MF}$ & $\omega_{\mathrm{cg}}$ \\
\hline 0 & $\infty$ & $\infty$ & 41.9424 & 2.2109 \\
\hline 0.05 & 20.6876 & 11.1185 & 35.6086 & 2.2109 \\
\hline 0.1 & 14.4302 & 6.9022 & 29.2747 & 2.2109 \\
\hline 0.15 & 10.3458 & 4.9458 & 22.9408 & 2.2109 \\
\hline 0.2 & 7.0076 & 3.7666 & 16.6070 & 2.2109 \\
\hline 0.25 & 4.0492 & 2.9846 & 10.2731 & 2.2109 \\
\hline 0.3 & 1.4434 & 2.4546 & 3.9393 & 2.2109 \\
\hline 0.35 & -0.8089 & 2.0871 & -2.3946 & 2.2109 \\
\hline 0.4 & -2.7154 & 1.8264 & -8.7284 & 2.2109 \\
\hline
\end{tabular}

Se aprecia de la Figura 6 (y de los resultados mostrados en la Tabla II) que el retardo adicionado en el lazo, disminuye el margen de fase y el margen de ganancia llegándose a situaciones inestables.

\section{PRUEBAS EXPERIMENTALES}

A. Prueba experimental con planta equivalente de primer orden con diferentes retardos.

Se experimentó con una planta de la cual luego de un procedimiento de identificación analógica [6], se obtuvo el siguiente modelo que la representa: 


$$
\begin{aligned}
& \mathrm{G}(\mathrm{s})=\frac{1.146 \mathrm{x} \mathbb{0}^{4}}{\mathrm{~s}^{2}+268.7 \mathrm{~s}+7641} \mathrm{e}^{-0.00119 \mathrm{~s}} \\
& \mathrm{G}(\mathrm{s})=\frac{1.146 \mathrm{x} \mathbb{0}^{4}}{(\mathrm{~s}+236.4)_{(\mathrm{s}+\mathbf{z})} \mathrm{e}^{-0.00119 \mathrm{~s}}}
\end{aligned}
$$

De acuerdo a (2), se obtiene para esta planta una constante de tiempo mayor $\mathrm{T}_{1}=0.0309 \mathrm{~s}$. y una constante de tiempo menor $\mathrm{T}_{2}=0.0042 \mathrm{~s}$. El tiempo de retardo es $m=0.00119 \mathrm{~s}$.

De acuerdo a (3) se obtiene la relación $\rho=\mathrm{T}_{2} / \mathrm{T}_{1}=$ 0.1359 y la relación $\mathrm{m} / \mathrm{t}_{1}=0.0388$. Así, el efecto de $\mathrm{T}_{2}$ y $\mathrm{m}$ son de menor consideración respecto a $\mathrm{T}_{1}$. Estos resultados significan que el sistema se comporta en forma efectiva como un sistema de primer orden. Para este sistema se obtiene como ganancia de la planta $\mathrm{K}_{\mathrm{G}}=1.5$. Para esa ganancia y $s_{1}=-1 / T_{1}=-32.3625$, el controlador PID dado por (20) es el siguiente:

$$
\mathrm{G}_{\text {Creal }}(\mathrm{s})=1.6667 \frac{(\mathrm{s}+\mathscr{D} .0)^{2}}{\mathrm{~s}(\mathrm{~s}+485.4)}
$$

Se implementó el sistema de lazo cerrado que representa la Figura 2 para el control de la planta utilizando el controlador PID dado por (27). Se aplicó una entrada de lazo cerrado tipo escalón y se registraron las características de la respuesta de lazo cerrado de la salida para diferentes tiempos de retardo de la planta no considerados en el diseño del controlador. En la Tabla IV se muestran los resultados de los parámetros dinámicos de la respuesta como son el tiempo de establecimiento $t_{s}$ como el valor de porcentaje de sobreimpulso $\mathrm{M}_{\mathrm{p}}$. En todos los casos en estado estacionario se anuló el error.

Tabla IV. Parámetros de respuesta al escalon en lazo cerrado del control de planta equivalente de primer orden para diferentes tiempos de retardo.

\begin{tabular}{|c|c|c|}
\hline $\mathrm{m} / \mathrm{T}_{1}$ & $\mathrm{M}_{\mathrm{p}}(\%)$ & $\mathrm{t}_{\mathrm{s}}(\mathrm{sg})$. \\
\hline 0.00 & 8.04 & 0.0605 \\
\hline 0.05 & 27.56 & 0.060 \\
\hline 0.10 & 66.01 & 0.1332 \\
\hline 0.15 & 109.35 & 2.80 \\
\hline 0.20 & \multicolumn{2}{|c|}{ Inestable } \\
\hline 0.25 & \multicolumn{2}{|c|}{ Inestable } \\
\hline
\end{tabular}

Fuente: Elaboración propia.

La Figura 7 muestra la respuesta medida en la salida para la planta anterior sin retardo para una entrada escalón de amplitud 0.8 voltios.

La Figura 8 muestra la respuesta medida en la salida para la planta anterior con un retardo de la planta efectivo de $\mathrm{m} / \mathrm{t}_{1}=0.15$, para una entrada escalón de amplitud 0.8 voltios.
B. Prueba experimental con planta equivalente de segundo orden sobreamortiguada representativa con diferentes retardos.

Se experimentó con una planta que luego de un procedimiento de identificación analógico se obtuvo el siguiente modelo que la representa:

$$
\begin{aligned}
& G(s)=\frac{1.334 x 0^{4}}{s^{2}+196.2 s+8894} e^{-0.000768 s} \\
& G(s)=\frac{1.334 x 00^{4}}{(s+125.1(s+1.1)} e^{-0.000768 s}
\end{aligned}
$$

Figura 7. Respuesta de lazo cerrado a entrada escalón de planta equivalente de primer orden sin retardo.

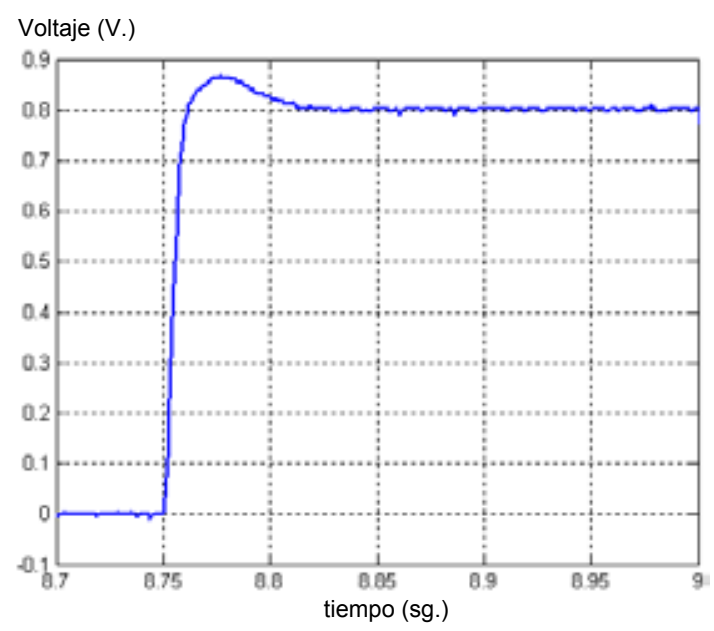

Figura 8. Respuesta de lazo cerrado a entrada escalón de planta equivalente de primer orden con retardo efectivo de $\mathrm{m} / \mathrm{T}_{1}=0.15$.

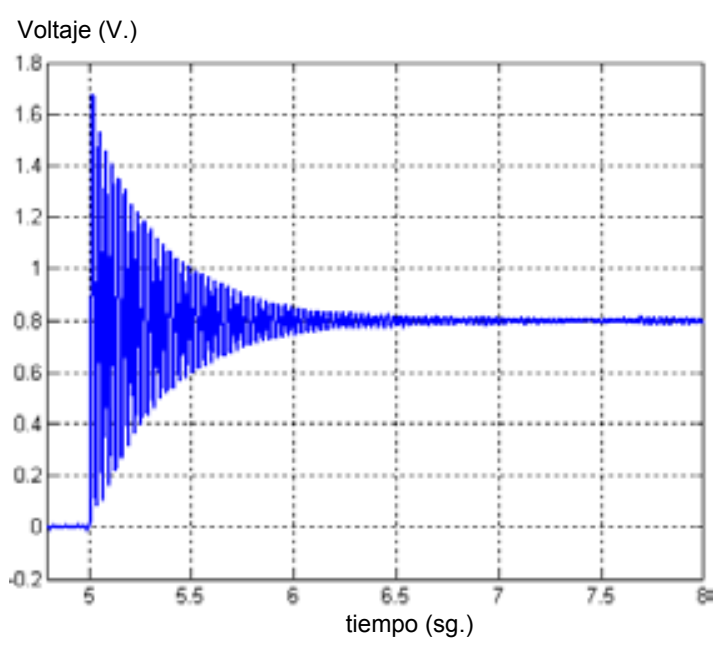


De acuerdo a (2), se obtiene para esta planta una constante de tiempo mayor $\mathrm{T}_{1}=0.0141 \mathrm{~s}$. y una constante de tiempo menor $\mathrm{T}_{2}=0.008 \mathrm{~s}$. El tiempo de retardo es $m=0.000768 \mathrm{~s}$. Se obtuvo la relación $\rho=T_{2} /$ $\mathrm{T}_{1}=0.5686$ y la relación $\mathrm{m} / \mathrm{T}_{1}=0.0546$. El efecto del retardo es despreciable se trata de un sistema sobreamortiguado de segundo orden. Para este sistema se obtiene como ganancia de la planta $\mathrm{K}_{\mathrm{G}}=1.5$. Para esa y $s_{1}=-1 / T_{1}=-70.922$, el controlador PID dado por (21) es el siguiente :

$$
\mathrm{G}_{\text {Creal }}(\mathrm{s})=0 \frac{(\mathrm{s}+141.8)^{2}}{\mathrm{~s}(\mathrm{~s}+709.2)}
$$

Se implementó el sistema de lazo cerrado que representa la Figura 2 para el control de la planta (28) utilizando el controlador PID dado por (29). Se aplicó una entrada de lazo cerrado tipo escalón y se registraron las características de la respuesta de lazo cerrado de la salida para diferentes tiempos de retardo de la planta no considerados en el diseño del controlador. En la Tabla $V$ se muestran los resultados de los parámetros dinámicos de la respuesta como son el tiempo de establecimiento $t$, como el valor de porcentaje de sobreimpulso $M_{\mathrm{p}}$. En todos los casos en estado estacionario se anuló el error.

La Figura 9 muestra la respuesta medida en la salida para la planta (28) sin retardo para una entrada escalón de amplitud 1.0 voltio.

La Figura 10 muestra la respuesta medida en la salida para la planta (28) anterior con un retardo de la planta efectivo de $\mathrm{m} / \mathrm{T}_{1}=0.30$ para una entrada escalón de amplitud 1.0 voltio.

Tabla V. Parámetros de respuesta al escalon en lazo cerrado del control de planta de segundo orden sobreamortiguada para diferentes tiempos de retardo.

\begin{tabular}{|l|c|c|}
\hline $\mathrm{m} / \mathrm{T}_{1}$ & $\mathrm{M}_{\mathrm{p}}(\%)$ & $\mathrm{t}_{\mathrm{s}}(\mathrm{sg})$. \\
\hline 0.00 & 12.79 & 0.041 \\
\hline 0.05 & 18.16 & 0.0411 \\
\hline 0.10 & 30.37 & 0.0361 \\
\hline 0.15 & 46.97 & 0.0458 \\
\hline 0.20 & 64.06 & 0.0812 \\
\hline 0.25 & 85.06 & 0.1391 \\
\hline 0.30 & 107.03 & 0.456 \\
\hline 0.35 & \multicolumn{2}{|c|}{ Inestable } \\
\hline 0.40 & \multicolumn{2}{|c|}{ Inestable } \\
\hline
\end{tabular}

Fuente : Elaboración propia.
C. Prueba experimental con planta equivalente de segundo orden críticamente amortiguada representativa con diferentes retardos.

Se experimentó con una planta con el siguiente modelo identificado:

$$
\begin{aligned}
& G(s)=\frac{1.705 x 0^{4}}{s^{2}+213.5 s+1.137 \times 0^{4}} \\
& G(s)=\frac{1.705 \times 0^{4}}{(s+111.9) s+101.6)}
\end{aligned}
$$

Figura 9. Respuesta de lazo cerrado a entrada escalón de planta de segundo orden sobreamortiguada sin retardo.

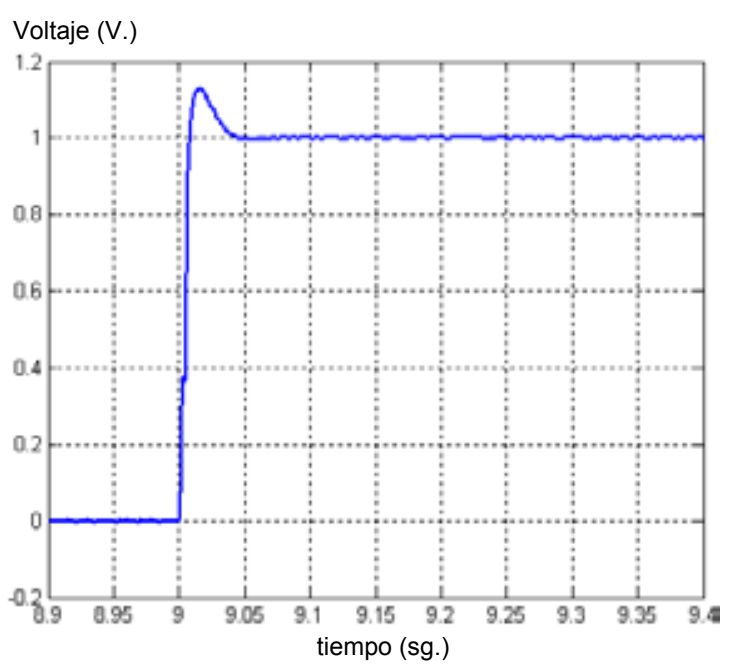

Figura 10. Respuesta de lazo cerrado a entrada escalón de planta de segundo orden sobreamortiguada con retardo efectivo de $\mathrm{m} / \mathrm{T}_{1}=0.30$.

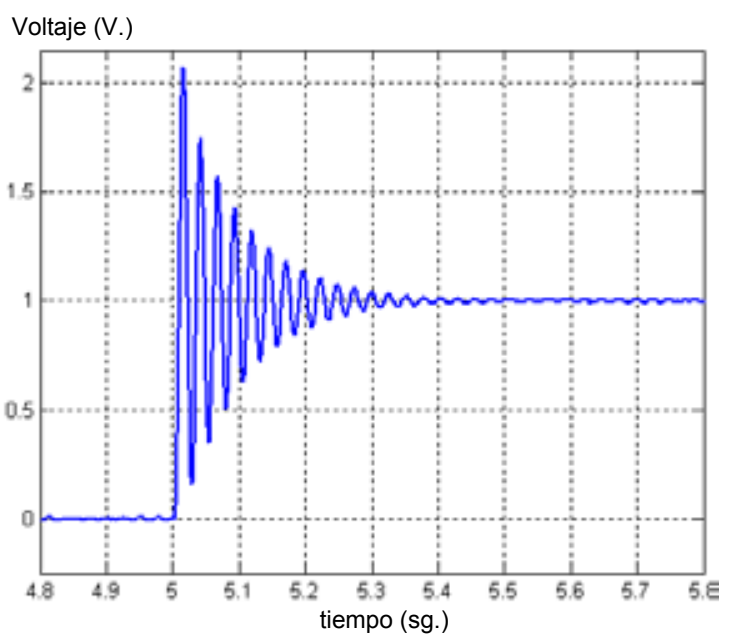


De acuerdo a (2), se obtiene para esta planta una constante de tiempo mayor $\mathrm{T}_{1}=0.0098 \mathrm{~s}$. y una constante de tiempo menor $\mathrm{T}_{2}=0.0089 \mathrm{~s}$. Se obtuvo la relación $\rho=\mathrm{T}_{2} / \mathrm{T}_{1}=0.9079$, se trata de un sistema aproximado a uno de segundo orden críticamente amortiguado. Para este sistema se obtiene como ganancia de la planta $\mathrm{K}_{\mathrm{G}}=1.5$. Para esa y $\mathrm{s}_{1}=-1 / \mathrm{T}_{1}=$ 102.0408, el controlador PID dado por (21) es el siguiente:

$$
\mathrm{G}_{\text {Creal }}(\mathrm{s})=\mathbb{0} \frac{(\mathrm{s}+204.1)^{2}}{\mathrm{~s}(\mathrm{~s}+1020)}
$$

Se implementó el sistema de lazo cerrado que representa la Figura 2 para el control de la planta (30) utilizando el controlador PID dado por (31). Se aplicó una entrada de lazo cerrado tipo escalón y se registraron las características de la respuesta de lazo cerrado de la salida para diferentes tiempos de retardo de la planta no considerados en el diseño del controlador. En la Tabla VI se muestran los resultados de los parámetros dinámicos de la respuesta como son el tiempo de establecimiento $t_{s}$,como el valor de porcentaje de sobreimpulso $M_{p}$. En todos los casos en estado estacionario se anuló el error.

Tabla VI. Parámetros de respuesta al escalon en lazo cerrado del control de planta de segundo orden críticamente amortiguada para diferentes tiempos de retardo.

\begin{tabular}{|c|c|c|}
\hline $\mathrm{m} / \mathrm{T}_{1}$ & $\mathrm{M}_{\mathrm{P}}(\%)$ & $\mathrm{t}_{\mathrm{s}}(\mathrm{sg})$. \\
\hline 0.00 & 25.00 & 0.042 \\
\hline 0.05 & 34.77 & 0.0438 \\
\hline 0.10 & 39.16 & 0.046 \\
\hline 0.15 & 52.34 & 0.044 \\
\hline 0.20 & 67.97 & 0.0528 \\
\hline 0.25 & 78.71 & 0.084 \\
\hline 0.30 & 97.75 & 0.138 \\
\hline 0.35 & 115.33 & 0.3981 \\
\hline 0.40 & \multicolumn{2}{|c|}{ Inestable } \\
\hline 0.45 & \multicolumn{2}{|c|}{ Inestable } \\
\hline
\end{tabular}

Fuente : Elaboración propia.

La Figura 11 muestra la respuesta medida en la salida para la planta (31) sin retardo para una entrada escalón de amplitud 1.0 voltio.
La Figura 12 muestra la respuesta medida en la salida para la planta (30) anterior con un retardo de la planta efectivo de $\mathrm{m} / \mathrm{\tau}_{1}=0.35$ para una entrada escalón de amplitud 1.0 voltio.

Figura 11. Respuesta de lazo cerrado a entrada escalón de planta de segundo orden críticamente amortiguada sin retardo.

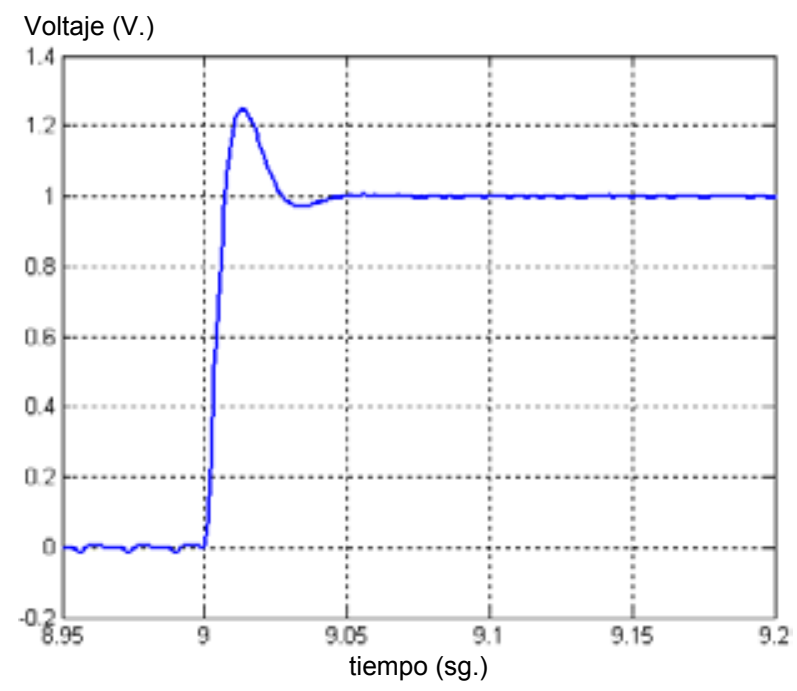

Figura 12. Respuesta de lazo cerrado a entrada escalón de planta de segundo orden críticamente amortiguada con retardo efectivo de $\mathrm{m} / \mathrm{T}_{1}=0.35$.

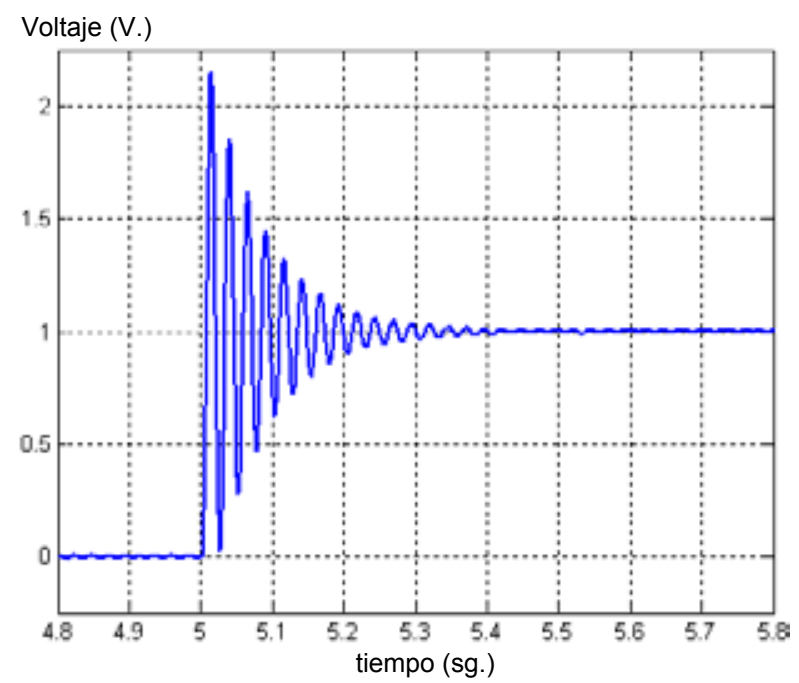




\section{CONCLUSIONES}

En las pruebas experimentales se confirma el hecho de que el aumento del retardo en la planta produce disminución del margen de fase y de ganancia de lazo cerrado, al punto de producir inestabilidad.

El efecto del retardo se aprecia en la respuesta dinámica en un incremento del sobreimpulso y del tiempo de establecimiento, así como en el incremento de las oscilaciones amortiguadas.

El sistema de primer orden es más sensible al aumento del retardo de la planta. El sistema de segundo orden sobreamortiguado es más sensible al aumento del retardo de la planta críticamente amortiguada.

Un leve aumento del retardo en $5 \%$ del retardo en relación a la constante de tiempo mayor $\mathrm{T}_{1}$ produce un incremento en el sobreimpulso que excede los estándares aceptados.

Se deben utilizar métodos de identificación que permitan obtener lo más cercano posible el tiempo de retardo de la planta, el cual necesariamente debe ser considerado en el diseño del controlador.

\section{REFERENCIAS BIBLIOGRÁFICAS}

[1] Martos, Marga. (2003). Control de procesos con grandes tiempos muertos. ESI Bilbao. http://www.disa.bi.ehu.es/spanish/asignaturas/17212/PRACTICA\%20IV.pdf.

[2] Hang C.C., Astrom K. J. \& Wang Q. G. (2002). Relay feedback auto-tuning of process controllers - a tutorial review, Journel of Process Control, 12, 143-162.http://www. disa.bi.ehu.es/spanish/asignaturas/17212/ PRACTICA\%20IV.pdf.

[3] Ogata, Katsuhiko. (1993). ingeniería de control moderna. México, Prentice-Hall Hispanoamericana, S.A.

[4] Vargas Tamani, Bruno. (2008). Diseño e implementacion de tarjeta electrónica de simulacion de procesos. Revista Electrónica UNMSM, 22.

[5] Franklin, G., Powell, D., \& Emami-Naeini, (1991). Control de sistemas dinámicos con retroalimentación. U.S.A., Addison-Wesley Iberoaméricana

[6] Alfaro, V. (2001). "Identificación de Procesos Sobreamortiguados Utilizando Técnicas de Lazo Abierto, Ingeniería", 1, 11-25. 